\title{
Usos del podcast para fines educativos. Mapeo sistemático de la literatura en WoS y Scopus (2014-2019)
}

\section{Uses of the podcast for educational purposes. Systematic mapping of the literature in WoS and Scopus (2014-2019)}

Iñaki Celaya. Universidad de Navarra. España.

icelaya@alumni.unav.es

$[\mathrm{CV}]$ (1) $\mathrm{G}$

María Soledad Ramírez-Montoya. Tecnológico de Monterrey. México.

solramirez@tec.mx

$[\underline{\mathrm{CV}}]$ (1) $\mathrm{C}$

Concepción Naval. Universidad de Navarra. España.

cnaval@unav.es

$[\mathrm{CV}](1) \mathrm{G}$

Elena Arbués. Universidad de Navarra. España.

earbues@unav.es

$[\mathrm{CV}](\mathrm{C})$

Este artículo cuenta con la colaboración del Grupo de Investigación consolidado en Educación, Ciudadanía y Carácter de la Universidad de Navarra (https://bit.ly/2ltO3qN); también participa el proyecto 266632 "Laboratorio Binacional para la Gestión Inteligente de la Sustentabilidad Energética y la Formación Tecnológica" ["Bi-National Laboratory on Smart Sustainable Energy Management and Technology Training”], apoyado por el CONACYT SENER Fondo para la sustentabilidad energética (Convenio:

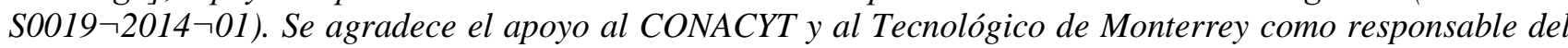
proyecto (https://bit.ly/2krVhLO). Asimismo, se agradece la financiación de la Asociación de Amigos de la Universidad de Navarra (https://bit.ly/2lCFNEN).

Fechas:

- Inicio de la investigación: 01/02/2019

- Término de la investigación: 01/08/2019

Cómo citar este artículo / Referencia normalizada

Celaya, I., Ramírez-Montoya, M. S., Naval, C. y Arbués, E. (2020). Usos del podcast para fines educativos. Mapeo sistemático de la literatura en WoS y Scopus (2014-2019). Revista Latina de Comunicación Social, (77), 179-201. https://www.doi.org/10.4185/RLCS-2020-1454

\section{RESUMEN}

Introducción: la investigación disponible sobre el aprovechamiento educativo del podcast de audio es escasa. Se revisó la literatura publicada (2014-2019) clasificando usos, contextos y categorías del podcast de audio con fines educativos, e identificando autores y revistas de referencia en el ámbito. Metodología: se aplicó el método de Mapeo Sistemático de la Literatura a una muestra de artículos indexados de acceso abierto en las bases de datos Web of Science y Scopus. El filtrado de la muestra se llevó a cabo de acuerdo con unos criterios de inclusión y exclusión. Resultados y conclusiones: 
se hallaron 81 artículos que destacan los principales usos educativos del podcast y coinciden en su utilidad para apoyar el aprendizaje en instituciones y entornos educativos formales, no formales e informales. Este trabajo brinda a investigadores, educadores e instituciones una línea base actualizada para seguir explorando las virtudes educativas del podcast.

PALABRAS CLAVE: podcast; e-learning; mapeo sistemático de la literatura; recursos educativos abiertos; educomunicación; educación.

\begin{abstract}
Introduction: There is little research available on the educational use of the audio podcast. The published literature (2014-2019) was reviewed, classifying uses, contexts, and categories of the audio podcast for educational purposes, and identifying authors and reference journals in the field. Methodology: The Systematic Mapping of Literature method was applied to a sample of open access articles indexed in the Web of Science and Scopus databases. The filtering of the sample was carried out according to inclusion and exclusion criteria. Results and conclusions: 81 articles were found that highlight the main educational uses of the podcast and coincide in its usefulness to support learning in formal, non-formal, and informal educational institutions and settings. This work provides researchers, educators, and institutions with an updated baseline to further explore the educational strengths of the podcast.
\end{abstract}

KEYWORDS: podcast; e-learning; systematic mapping of literature; open educational resources; educommunication; education.

\title{
CONTENIDOS
}

1. Introducción. 2. Método. 2.1. Preguntas de investigación. 2.2. Cadenas de búsqueda booleanas. 2.3. Criterios de inclusión y exclusión. 3. Resultados. 4. Discusión y conclusiones. 5. Referencias.

\section{Introducción}

La educomunicación estudia fenómenos que emanan de las sinergias entre comunicación y educación. No solo trata de educar a través de los medios de comunicación, sino que fomenta una educación encaminada a la gestión de la información, la tecnología relacionada con internet y los medios de comunicación masivos (Aguaded, 2005; 2011; 2012). La irrupción de la Web 2.0 de O’Reilly (2005) supuso un acicate para la participación de la ciudadanía en internet, contexto que según Naval y Arbués (2015) configura espacios privilegiados de participación. A partir de ello, se consolida lo que Jenkins, Purushotma, Werigel, Clinton y Robinson (2009) han denominado cultura participativa. En este contexto, se vuelve esencial la capacidad de discernir y valorar de forma crítica la ingente cantidad de información disponible. Atrás quedan conceptos como los de alfabetización informática y alfabetización informacional (Gallado, 2013; Bundy, 2004), centrados en el manejo de los medios en su dimensión más técnica. El paradigma de la educación mediática resulta más apropiado en tanto en cuanto considera aspectos relativos a los contenidos y su consumo. Como indican Naval, Serrano-Puche, Sádaba y Arbués (2016), la educación mediática es ya una prioridad, tanto del sistema educativo, como de los principales organismos internacionales. En esta línea, Khan (2009) ahonda en los retos de la educación mediática y establece que es crucial la colaboración entre gobiernos, empresas y sociedad civil para avanzar en este camino (p. 10).

La transformación de información en conocimiento se da a través de un proceso humano dirigido, una coordinación de competencias cognitivas que da sentido a los datos, para ordenarlos coherentemente, para que resulten útiles y puedan resolver problemas (Díaz, 2012). Es así como el constructo de sociedad de la información evoluciona al de sociedad del conocimiento (Castells, 
2006; Phillips, Hameed \& El Akhdary, 2017). Las personas que conforman la sociedad se apropian continuamente de elementos del medio, los procesan como conocimientos y se educan, lo que se conoce como aprendizaje informal (Esclapez, 2008).

Por otro lado, en el sistema educativo formal, los elementos mencionados se cristalizan a través de enfoques derivados del e-learning, el m-learning (aprendizaje a través de dispositivos móviles) y el u-learning (aprendizaje ubicuo), entre otros. En esa línea, Keegan (2005) destaca las oportunidades de aprendizaje latentes en las plataformas móviles -tabletas, teléfonos y sus servicios complementarios ligados a internet-, campo que se ha denominado m-learning. Por su parte, Burbules $(2012 ; 2014)$ y Zapata-Ros (2012) explican cómo el u-learning aprovecha la omnipresencia de aquellos medios y fuentes con potencial educativo que brinda la sociedad del conocimiento, los cuales superan muchas de las limitaciones de los sistemas de enseñanza tradicionales.

El movimiento de educación abierta busca superar las limitaciones de acceso y propiedad intelectual que presentan los recursos analógicos tradicionales (Blessinger \& Bliss, 2016). Unesco (2002) defiende la proliferación de nuevos Recursos Educativos Abiertos (REA), apoyados en las Tecnologías de la Información y de la Comunicación (TIC), con usos no comerciales. Se crean y aprovechan aquellos materiales o actividades educativas que permiten prácticas formativas de libre acceso. En consecuencia, D'Antoni (2009) apunta a que ciudadanos, educadores e instituciones han sido los principales agentes beneficiados.

Los avances más relevantes del movimiento educativo abierto han sido promover el contenido abierto de calidad, romper barreras para abrir los contenidos educativos y alentar a las personas en todo el mundo a usar los REA. Éstos, atendiendo a una taxonomía de cuatro etapas, van desde la producción de actividades y REA disponibles en internet, hasta la selección y uso de materiales a través de repositorios y conectores que actúan como infomediarios de los catálogos; así como la diseminación de prácticas abiertas en entornos académicos, gubernamentales, institucionales, ONG, academias, empresa y otras instancias, y prácticas de movilización que permiten ser integradas en acciones formativas (Ramírez-Montoya, 2018; Ramírez-Montoya y Burgos, 2012).

Es realmente interesante observar cómo actualmente la mayoría de medios con potencial educativo basados en las TIC escapan a categorizaciones puramente educativas o comunicativas, y adquieren una naturaleza dual. Es lo que ocurre con blogs, vlogs, wikis o podcasts, entre otros: son objetos educomunicativos. Entre ellos, el formato podcast, en particular, es un medio de comunicación digital de la era 2.0. De acuerdo con Sellas (2011) empieza a definirse en los primeros años 2000, fruto del intercambio de archivos de audio entre usuarios tecnófilos, pero toma entidad oficialmente en 2004, cuando el periodista de tecnología Ben Hammersley (2004) describe la consolidación de dichas prácticas en un artículo llamado Audible revolution. Mientras que popularmente se lo ha llamado «radio por internet», Gallego (2010) vincula la identidad del podcast a la sindicación automática vía protocolo RSS, que permite su distribución libre y gratuita por internet, así como su consumo en diferido bajo demanda del usuario.

El formato podcast puede contener solo audio o también vídeo asociado, en cuyo caso se denomina vídeo podcast o vodcast, que se asemeja al formato videoblog. El podcasting está viviendo un importante auge como medio de comunicación en los últimos años, donde predomina el audio. De hecho, es un análogo al fenómeno YouTube en el terreno del audio, pues recoge iniciativas amateurs a la vez que sirve de extensión a los medios de comunicación tradicionales, y son plataformas como Spotify o Apple Podcasts las que hacen las veces de escaparate. Estados Unidos es el país donde más podcasts se escuchan. De hecho, Edison Research (2019) concluye que 90 millones de estadounidenses, el 32\% de la población, habrían escuchado podcasts en el último mes. Los datos en 
España son más modestos, 350000 oyentes, un 1,2\% de los internautas, según el EGM (2017). Sin embargo, es un formato que muestra una tendencia de consumo al alza.

La comunidad educativa ha experimentado desde muy temprano con el formato podcast. La investigación sobre su aprovechamiento educativo se ha visto impulsada en sus inicios por proyectos universitarios como el Duke Digital Initiative, que en su edición de 2005 a 2006 exploraba el potencial de la producción y consumo de contenidos académicos en formatos de podcast de audio y de vídeo (Earp, Belanger, \& O`Brien, 2006). Borges (2009) señaló ventajas cognitivas debido a su uso en el aula y una mayor implicación, autogestión, disponibilidad del material, continuidad en el estudio, comprensión, y reducción de la ansiedad (pp. 35-36); enfatizaba asimismo que las clases grabadas o lecturecasting es solo una de sus posibles aplicaciones (p.43). De hecho, la organización EDUCAUSE, que promueve la innovación en educación superior a través de las TIC, se sirve del podcast como medio de difusión de entrevistas sobre innovación, buenas prácticas educativas y formación docente. También en esta línea, pero en el ámbito informal, se ha estudiado el podcast como contenedor y difusor de divulgación científica (de-Lara-González \& del-Campo-Cañizares, 2018), mientras que autores como Forbes \& Khoo (2015) se han centrado en su utilidad para impartir formación en modo no presencial.

En cuanto a metaestudios sobre el podcasting, destaca la revisión que Hew (2009) presentó hace una década, además de trabajos más recientes enfocados hacia los ámbitos de la comunicación radiofónica (Arribas, Gutiérrez, Fragoso \& Arcos, 2018), formación en medicina de emergencia (Paterson, Thoma, Milne, Lin \& Chan, 2015), aprendizaje de idiomas (Hasan \& Hoon, 2013; Lomicka \& Lord, 2011) y educación superior (McGarr, 2009). Estos trabajos son muy específicos y no abordan los usos educativos del podcast de forma agregada y multidisciplinar. En consecuencia, consideramos novedosa una revisión actualizada que explore la literatura relativa al podcast con fines educativos en todas las disciplinas, para obtener información sobre el estado actual del objeto de estudio.

Este trabajo presenta los resultados obtenidos a partir de un mapeo sistemático de la literatura, que se llevó a cabo a principios de 2019, con el fin de conocer los usos educativos que se le han dado al podcast de audio. Para ello, se localizó la literatura comprendida entre enero de 2014 y febrero de 2019, en las principales bases de datos (Scopus y Web of Science), abarcando un período de cinco años. Se detalla el método aplicado en el estudio y los resultados obtenidos, así como el análisis de los datos, que actualiza el estado del arte sobre el podcast educativo. Las conclusiones apuntan tanto a la comunidad educativa como a la académica, ahondando en la virtualidad educativa del podcasting, y también alumbrando nuevas líneas de investigación que pueden ser útiles para desarrollar futuras investigaciones en la materia.

\section{Método}

El Mapeo Sistemático de la Literatura es un método de investigación que permite trazar la evidencia en un dominio con un alto nivel de granularidad. Esto ayuda a indentificar grupos de evidencia y desiertos de evidencia de cara a desarrollar futuras revisiones de la literatura y estudios primarios. (Kitchenham \& Charters, 2007; Kitchenham, Pretorius, Budgen, Brereton, Turner, Niazi, \& Linkman, 2010). Es común utilizar el mapeo como fase previa (descriptiva) a una revisión sistemática (analítica), lo que da mayor contextualización y estructura al objeto de estudio (CASCADE, 2012).

\subsection{Preguntas de investigación}

En primer lugar, tal y como sugieren las colaboraciones de Kroll, Richardson, Prikladnicki y Audy (2018) y Petersen, Vakkalanka y Kuzniarz (2015), se establecieron una serie de preguntas de investigación (RQ), para enfocar la estrategia de búsqueda. 
- RQ1: ¿Cuántos estudios de acceso abierto sobre el tratamiento educativo del podcast de audio hay en las bases de datos Scopus y Web of Science (WoS) entre enero de 2014 y febrero de 2019 ?

○ RQ1.1: ¿Qué métodos de investigación se aplicaron en los estudios?

○ RQ1.2: ¿Cuál es la distribución geográfica de los autores que más publicaron en el área?

○ RQ1.3: ¿Cuáles son los artículos más citados?

○ RQ1.4: ¿Cuáles son las revistas que más publicaron sobre la temática?

- RQ2: ¿En qué contextos se desarrollaron los estudios?

- RQ3: ¿Cuáles son las categorías temáticas en las que se estructuró el uso educativo del podcast de audio?

En segundo lugar, para contestar a las RQ planteadas, se diseñó una estrategia de búsqueda que se limitó a artículos de revistas indexadas en las bases de datos Scopus y WoS (SCI-E; SSCI; A\&HCI; ESCI), las cuales se escogieron atendiendo a su vasta colección y dilatada reputación en el ámbito científico. Los ejes de la estrategia de selección de artículos fueron el establecimiento de criterios de inclusión, duplicidad y exclusión, aplicados a unas cadenas de búsqueda booleanas.

\subsection{Cadenas de búsqueda booleanas}

Se utilizaron cadenas de búsqueda compuestas por expresiones booleanas basadas en las palabras clave podcast, online radio, profcast, lecturecasting, education y train. Con esos parámetros se obtuvieron 2447 resultados iniciales: 1659 en Scopus y 788 en WoS. Los resultados brutos fueron obtenidos a partir de las cadenas de búsqueda que refleja la tabla 1.

Tabla 1. Cadenas de búsqueda.

\begin{tabular}{ll}
\hline & TITLE-ABS-KEY (“online radio” OR podcast* OR profcast OR lecturecasting) AND \\
(educ* OR train*) \\
Limitado a: (LIMIT-TO (PUBSTAGE, "final")) AND (LIMIT-TO (ACCESSTYPE \\
(Open Access)) AND LIMIT-TO (PUBYEAR, 2019) OR LIMIT-TO (PUBYEAR, \\
2018) OR LIMIT-TO (PUBYEAR, 2017) OR LIMIT-TO (PUBYEAR, 2016) OR \\
LIMIT-TO (PUBYEAR, 2015) OR LIMIT-TO (PUBYEAR, 2014)) AND (LIMIT-TO \\
(DOCTYPE, "article" )) AND (LIMIT-TO (SRCTYP, "journal")) \\
\hline \\
TS=((“online radio” OR podcast* OR profcast OR lecturecasting) AND (educ* OR \\
train*) \\
Refinado por: PUBLICATION YEARS: (2019 OR 2017 OR 2015 OR 2014 OR 2018 \\
OR 2016) AND Open Access: (OPEN ACCESS) AND DOCUMENT TYPES: \\
(ARTICLE) \\
Indexes=SCI-EXPANDED, SSCI, A\&HCI, ESCI Timespan=2014-2019
\end{tabular}

Fuente: elaboración propia.

\subsection{Criterios de inclusión y exclusión}

Los criterios de inclusión y exclusión propuestos para realizar el filtrado de la muestra original se decidieron atendiendo al pragmatismo, buscando un equilibrio razonable entre cantidad y calidad. De acuerdo con la tabla 2, se excluyeron aquellos artículos provenientes de revistas cerradas por la dificultad de acceder al texto completo. Atendiendo a Melero y Hernández-San-Miguel (2014) y García-Peñalvo (2017) la ciencia abierta facilita la circulación de los datos de investigación entre los investigadores, y garantiza la reproducibilidad de los estudios. Además, estudios como el de Breugelmans, Roberge, Tippett, Durning, Struck y Makanga (2018), McKiernan et al. (2016), 
Piwowar et al. (2018) y Wang, Liu, Mao y Fang (2015) sugieren que los artículos de acceso abierto suelen obtener un mayor impacto científico medido en citas bibliográficas. Por otro lado, se decidió no filtrar por disciplina, puesto que el podcast se ha utilizado con fines educativos en áreas ajenas a la educación. No se limitó la variable idioma. Sí que se descartaron estudios que usaran podcasts de vídeo. El vodcast aparece en múltiples trabajos de investigación educativa donde, muchas veces, se engloba audio y vídeo bajo el mismo concepto de podcast, sin distinción. Esa disparidad conceptual puede llevar a confusión pues se trata de recursos diferentes, como apunta Brabazon (2016), que además señala que el valor añadido del vídeo en la asimilación del contenido es discutible (p. 135). Estas diferencias y el hecho de que el vídeo en educación haya estado de moda en los últimos años nos llevan a focalizar la atención esta vez en el podcast de audio.

Tabla 2. Criterios de inclusión y exclusión.

\begin{tabular}{ll}
\hline Criterios de inclusión & Criterios de exclusión \\
\hline $\begin{array}{l}\text { Artículos con las palabras clave "podcast*”, } \\
\text { "profcast", "lecturecasting", "educ*”, "train*”. }\end{array}$ & Artículos sin conexión educativa. \\
$\begin{array}{ll}\text { Que estén en las bases de datos Scopus y WoS } & \text { Trabajos que se centran en podcasts de video } \\
\text { ( } \text { (vodcasts). }\end{array}$ \\
$\begin{array}{l}\text { Artículos comprendidos entre enero de 2014 y } \\
\text { febrero de 2019. }\end{array}$ & $\begin{array}{l}\text { Referencias que son en sí mismos podcasts (audio- } \\
\text { artículos) y no artículos de revista. }\end{array}$ \\
Todas las disciplinas e idiomas. & Artículos de acceso cerrado. \\
Artículos de acceso abierto. & \\
\hline
\end{tabular}

Fuente: elaboración propia.

Una vez filtrada la búsqueda inicial de acuerdo con los criterios de inclusión, quedaron 169 ítems: 76 en Scopus y 93 en WoS. Entonces se eliminaron los ítems duplicados. Para ello, se dio prioridad a la base de datos Scopus y, en consecuencia, se descartaron 34 ítems duplicados de WoS. Posteriormente, se leyeron los títulos y el abstract de cada uno de los artículos restantes. Se rechazaron 54 artículos más después de aplicar los criterios de exclusión. La figura 1 muestra un diagrama con el proceso de filtrado realizado hasta obtener la muestra final.

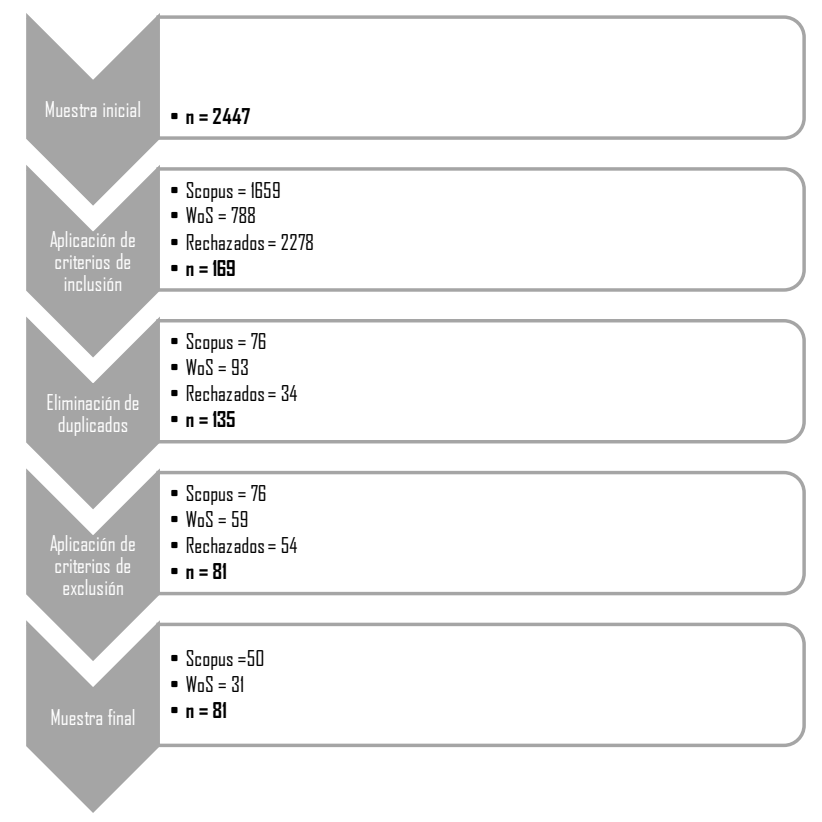

Figura 1: diagrama con los pasos de filtrado de la muestra.

Fuente: elaboración propia. 


\section{Resultados}

RQ1 - ¿Cuántos estudios de acceso abierto sobre el tratamiento educativo del podcast de audio hay en las bases de datos Scopus y WoS entre enero de 2014 y febrero de 2019? Tras filtrar por completo la muestra se obtuvieron un total de 81 artículos relacionados con el uso educativo del podcast los cuales se incluyen en la tabla 3.

Tabla 3. Muestra final de artículos sobre el uso educativo del podcast.

\begin{tabular}{|c|c|}
\hline Ref. & tor, título y revista \\
\hline 1 & $\begin{array}{l}\text { Abdoli-Sejzi, A., Aris, B., Ahmad, M. H., \& Rosli, M. S. (2015). The relationship between web } \\
2.0 \text { technologies and students achievement in virtual university. International Education } \\
\text { Studies, } 8(13), 67-72 \text {. Recuperado de doi: } 10.5539 / \text { ies.v8n13p67 }\end{array}$ \\
\hline 2 & $\begin{array}{l}\text { Álvarez-Moreno, M. A., \& Vásquez-Carvajal, S. C. (2015). Radio y cultura: Una propuesta de } \\
\text { radio ciudadana en Internet. Palabra Clave, 18(2), 475-498. Recuperado de } \\
\text { doi:10.5294/pacla.2015.18.2.7 }\end{array}$ \\
\hline 3 & $\begin{array}{l}\text { Ausín, V., Abella, V., Delgado, V., \& Hortigüela, D. (2016). Project-based learning through } \\
\text { ICT. An experience of teaching innovation from university classrooms [Aprendizaje basado en } \\
\text { proyectos a través de las TIC. Una experiencia de innovación docente desde las aulas } \\
\text { universitarias]. Formación Universitaria, 9(3), 31-38. Recuperado de doi: } 10.4067 / \text { S0718- } \\
50062016000300005\end{array}$ \\
\hline 4 & $\begin{array}{l}\text { Barber, J. F. (2016). Digital storytelling: New opportunities for humanities scholarship and } \\
\text { pedagogy. Cogent Arts \& } \quad \text { Humanities, 3(1). Recuperado de doi: } \\
\text { 10.1080/23311983.2016.1181037 }\end{array}$ \\
\hline 5 & $\begin{array}{l}\text { Bednarczyk, J., Pauls, M., Fridfinnson, J., \& Weldon, E. (2014). Characteristics of evidence- } \\
\text { based medicine training in Royal College of Physicians and Surgeons of Canada emergency } \\
\text { medicine residencies - a national survey of program directors. BMC Medical Education, 14(1). } \\
\text { Recuperado de doi: } 10.1186 / 1472-6920-14-57\end{array}$ \\
\hline 6 & $\begin{array}{l}\text { Blomgren, C. (2018). OER Awareness and Use: The Affinity Between Higher Education and K- } \\
\text { 12. International Review of Research in Open and Distributed Learning, 19(2), 55-70. } \\
\text { Recuperado de doi: 10.19173/irrodl.v19i2.3431 }\end{array}$ \\
\hline 7 & $\begin{array}{l}\text { Bounds, R., \& Boone, S. (2018). The Flipped Journal Club. Western Journal of Emergency } \\
\text { Medicine, 19(1), 23-27. Recuperado de doi: 10.5811/westjem.2017.11.34465 }\end{array}$ \\
\hline 8 & $\begin{array}{l}\text { Brites, M. J., Santos, S. C., Jorge, A., \& Catalão, D. (2017). Ferramentas jornalísticas na } \\
\text { educação: Uma rádio online para jovens. Sociologia, Problemas e Práticas, (84), 81-100. } \\
\text { Recuperado de doi: } 10.7458 / \text { SPP2017846102 }\end{array}$ \\
\hline 9 & $\begin{array}{l}\text { Bucher, J., Donovan, C., \& McCoy, J. (2018). EMS providers do not use FOAM for education. } \\
\text { International Journal of Emergency Medicine, 11(1). Recuperado de doi: 10.1186/s12245-018- } \\
0189-4\end{array}$ \\
\hline 10 & $\begin{array}{l}\text { Chan, T. M., Thoma, B., Krishnan, K., Lin, M., Carpenter, C. R., Astin, M., \& Kulasegaram, K. } \\
\text { (2016). Derivation of Two Critical Appraisal Scores for Trainees to Evaluate Online } \\
\text { Educational Resources: A METRIQ Study. Western Journal of Emergency Medicine, 17(5), } \\
\text { 574-584. Recuperado de doi: 10.5811/westjem.2016.6.30825 }\end{array}$ \\
\hline
\end{tabular}

11 Chin, A., Helman, A., \& Chan, T. M. (2017). Podcast use in undergraduate medical education. Cureus, 9(12). Recuperado de https://dx.doi.org/10.7759\%2Fcureus.1930

Cortés-Fuentes, J. -A., \& Correyero-Ruiz, B. (2017). Radio and social empowerment. Uses and the rewards gained of radio workshops for individuals with intellectual or developmental

12 disabilities [Radio y empoderamiento social. Usos y gratificaciónes del taller de radio para las personas con discapacidad intelectual o del desarrollo]. Profesional de la Información, 26(1), 55-65. Recuperado de doi: 10.3145/epi.2017.ene.06

Da Costa Miranda De Carvalho, F. B., De Lima, C. P., Dutra, A., Da Rosa, V. F., \& De Oliveira, J. (2018). Use of podcast and webquest resources in the study of assessment in early

13 childhood education [Uso de recursos podcast e webquest no estudo do tema avaliação na educação infantil]. Texto Livre, 11(2), 192-205. Recuperado de doi: 10.17851/19833652.11.2.192-205 
Dantas-Queiroz, M. V., Wentzel, L. C. P., \& Queiroz, L. L. (2018). Science communication podcasting in Brazil: the potential and challenges depicted by two podcasts. Anais da Academia Brasileira de Ciencias, 90(2), 1891-1901. Recuperado de doi: 10.1590/00013765201820170431

Day, L., Cunsolo, A., Castleden, H., Martin, D., Hart, C., Anaviapik-Soucie, T., Russell, G., Paul, C., Dewey, C., \& Harper, S. L. (2017). The expanding digital media landscape of

15 qualitative and decolonizing research: examining collaborative podcasting as a research method. Media Tropes, 7(1), 203-228. $\quad$ Recuperado de https://mediatropes.com/index.php/Mediatropes/article/view/28320

Dobinson, T. J., \& Bogachenko, T. (2018). Issues arising from the use of university ilectures case study of one Australian campus. Australian Journal of Teacher Education, 43(2), 100-115. Recuperado de doi: 10.14221/ajte.2018v43n2.6

Doerr-Stevens, C., \& Buckley-Marudas, M. (2019). Hearing Knowledge into Action:

17 Mobilizing Sound for Multicultural Imaginaries. International Journal of Multicultural Education, 21(1), 105-124. Recuperado de http://dx.doi.org/10.18251/ijme.v21i1.1735

Dos Reis, S. C., \& Gomes, A. F. (2014). Podcasts for teaching English as a Foreign Language:

18 Analysis and digital literacy practice [Podcasts para o ensino de Língua Inglesa: Análise e prática de Letramento Digital1]. Calidoscopio, 12(3), 367-379. Recuperado de doi: 10.4013/cld.2014.123.11

19 Drigas, A. S., \& Angelidakis, P. (2017). Mobile applications within education: An overview of application paradigms in specific categories. International Journal of Interactive Mobile Technologies, 11(4), 17-29. Recuperado de doi: 10.3991/ijim.v11i4.6589

Fouz-González, J. (2018). Podcast-based pronunciation training: Enhancing FL learners'

20 perception and production of fossilised segmental features. ReCALL, 31(2), 150-169. Recuperado de https://doi.org/10.1017/S0958344018000174

Freire, E. P. A. (2015). School podcast's cooperative potentials in a freinet's perspective [Potenciais cooperativos do podcast escolar por uma perspectiva freinetiana]. Revista Brasileira de Educacao, 20(63), 1033-1056. Recuperado de doi: 10.1590/S1413-24782015206312

Freire, E. P. A. (2016). Communication/education by Paulo Freire in Brazilian "Podosfera".

22 Comunicaçoes, 23(2), 29-52. Recuperado de http://dx.doi.org/10.15600/2238$121 \mathrm{X} /$ comunicacoes. $23 \mathrm{n} 2 \mathrm{p} 29-52$

García-Peinazo, D. (2018). A la parrilla suena mejor: El podcast como estrategia didáctica basada en el ABP para el análisis y la gestión de eventos en la asignatura Historia de la Música /

23 A la parrilla suena mejor: Podcast as a Project-Based-Learning Didactic Tool for Music History and Event Management. Tercio Creciente, 8(1), 73-84. Recuperado de https://doi.org/10.17561/rtc.n15.5

Gillie, M., Dahli, R., Saunders, F. C., \& Gibson, A. (2017).Use of rich-media resources by engineering undergraduates. European Journal of Engineering Education, 42(6), 1496-1511. Recuperado de https://doi.org/10.1080/03043797.2017.1306488

Gonchar, I. A., \& Popova, T. I. (2018). The Structure of Online Educational Resource on

25 Russian as Foreign Language: Approaches to Modelling Communication. Philological Class, 4(54), 78-85. Recuperado de doi: 10.26710/fk18-04-11

Grau-Ibarra, K., \& Villarreal-Orias, J. (2017). Relevance of eight radio programs (or podcasts)

26 as teaching material in distance education in an introductory subject to natural resource management. Revista Electrónica Calidad en la Educación Superior, 8(1), 335-357. Recuperado de https://doi.org/10.22458/caes.v8i1.1796

Grock, A., Rezaie, S., Swaminathan, A., Min, A., Shah, K. H., \& Lin, M. (2017). Blog and Podcast Watch: Orthopedic Emergencies. Western Journal of Emergency Medicine, 18(3), 531538. Recuperado de doi: 10.5811/westjem.2017.1.33197

Grock, A., Morley, E. J., Roppolo, L., Khadpe, J., Ankel, F., \& Lin, M. (2017). Blog and

28 Podcast Watch: Cutaneous Emergencies. Western Journal of Emergency Medicine, 18(2), 288292. Recuperado de doi: 10.5811/westjem.2016.11.32092

Grock, A., Joshi, N., Swaminathan, A., Rezaie, S., Gaafary, C., \& Lin, M. (2016). Blog and

29 Podcast Watch: Neurologic Emergencies. Western Journal of Emergency Medicine, 17(6), 726733. Recuperado de doi: 10.5811/westjem.2016.9.31010

Ikonne, U., Campbell, A. M., Whelihan, K. E., Bay, R. C., \& Lewis, J. H. (2018). Exodus from the classroom: student perceptions, lecture capture technology, and the inception of on-demand preclinical medical education. Journal of the American Osteopathic Association, 118(12), 813823. Recuperado de https://doi.org/10.7556/jaoa.2018.174 
Impey, C. D., Wenger, M. C., \& Austin, C. L. (2015). Astronomy for Astronomical Numbers: A Worldwide Massive Open Online Class. International Review of Research in Open and Distance Learning, 16(1), 57-79. Recuperado de doi: 10.19173/irrodl.v16i1.1983

Joshi, N., Morley, E. J., Taira, T., Branzetti, J., \& Grock, A. (2017). ALiEM Blog and Podcast

32 Watch: Procedures in Emergency Medicine. Western Journal of Emergency Medicine, 18(6), 1128-1134. Recuperado de doi: 10.5811/westjem.2017.8.34844

33 Kinash, S., Knight, D., \& McLean, M. (2015). Does Digital Scholarship through Online Recuperado de https://www.j-ets.net/ETS/journals/18_2/10.pdf

Kornieva, Z. M., \& Vashchylo, O. V. (2019). Efficiency of teaching English monologue 34 production to future mechanical engineers by means of podcasting. Information Technologies and Learning Tools, 74(6), 71-83. Recuperado de https://doi.org/10.33407/itlt.v74i6.3120

Krueger, S. (2018). LIS Students at a Japanese University Use Smartphones for Social Communication more often than for Educational Purposes. Evidence Based Library and Information Practice, 13(3), 97-99. Recuperado de doi: 10.18438/eblip29412

Kwary, D. A., \& Fauzie, S. (2018). Students' achievement and opinions on the implementation

36 of e-learning for phonetics and phonology lectures at Airlangga University. Educacao $e$ Pesquisa, 44(1). Recuperado de doi: 10.1590/S1678-4634201710173240

Langdorf, M. I., Anderson, C. L., Navarro, R. E., Strom, S., McCoy, C. E., Youm, J., \& YpmaWong, M. F. (2018). Comparing the results of written testing for advanced cardiac life support teaching using team-based learning and the "Flipped Classroom" strategy. Cureus, 10(5). Recuperado de https://dx.doi.org/10.7759\%2Fcureus.2574

LeRouge, C., Van Slyke, C., Seale, D., \& Wright, K. (2014). Baby Boomers' Adoption of 38 Consumer Health Technologies: Survey on Readiness and Barriers. Journal of Medical Internet Research, 16(9). Recuperado de doi: 10.2196/jmir.3049

Levesley, A., Paxton, S., Collins, R., Baker, A., \& Knight, C. (2014). Engaging students with plant science: the Plant Science TREE. New Phytologist, 203(4), 1041-1048. Recuperado de doi: 10.1111/nph.12905

Lien, K., Chin, A., Helman, A., \& Chan, T. M. (2018). A randomized comparative trial of the knowledge retention and usage conditions in undergraduate medical students using podcasts and blog posts. Cureus, 10(1). Recuperado de https://dx.doi.org/10.7759\%2Fcureus.2065 Lin, K., Chan, K., Mohindra, R., Milne, K., Thoma, B., \& Bond, C. (2017). SGEM hot off the press: Computer provider order entry (CPOE) and emergency department flow. Canadian Journal of Emergency Medicine, 19(2), 147-153. Recuperado de https://doi.org/10.1017/cem.2017.7

Lin, M., Thoma, B., Trueger, N. S., Ankel, F., Sherbino, J., \& Chan, T. (2015). Quality indicators for blogs and podcasts used in medical education: modified Delphi consensus recommendations by an international cohort of health professions educators. Postgraduate Medical Journal, 91(1080), 546-550. Recuperado de doi: 10.1136/postgradmedj-2014-133230

Mallin, M., Schlein, S., Doctor, S., Stroud, S., Dawson, M., \& Fix, M. (2014). A survey of the

43 current utilization of asynchronous education among emergency medicine residents in the United States. Academic Medicine, 89(4), 598-601. Recuperado de doi: 10.1097/ACM.0000000000000170

Melkers, J., Hicks, D., Rosenblum, S., Isett, K. R., \& Elliott, J. (2017). Dental Blogs, Podcasts,

44 and Associated Social Media: Descriptive Mapping and Analysis. Journal of Medical Internet Research, 19(7). Recuperado de doi: 10.2196/jmir.7868

Meyliana, Hidayanto, A. N., \& Budiardjo, E. K. (2015). Evaluation of Social Media Channel

45 Preference for Student Engagement Improvement in Universities Using Entropy and Topsis Method. Journal of Industrial Engineering and Management-JIEM, 8(5), 1676-1697. Recuperado de doi: 10.3926/jiem.1652

Middleton, A. (2016). Reconsidering the role of recorded audio as a rich, flexible and engaging

46 learning space. Research in Learning Technology, 24, 28035. Recuperado de doi: 10.3402/rlt.v24.28035

Msonde, S. E., \& Van Aalst, J. (2017). Designing for interaction, thinking and academic

47 achievement in a Tanzanian undergraduate chemistry course. ETR\&D-Educational Technology Research and Development, 65(5), 1389-1413. Recuperado de doi: 10.1007/s11423-017-9531-4 Naseri, S., \& Motallebzadeh, K. (2016). Podcasts: A Factor to Improve Iranian EFL Learner'

48 Self-Regulation Ability and Use of Technology. Educational Technology \& Society, 19(2), 328-339. Recuperado de https://www.j-ets.net/ETS/journals/19_2/24.pdf 
Nwosu, A. C., Monnery, D., Reid, V. L., \& Chapman, L. (2017). Use of podcast technology to facilitate education, communication and dissemination in palliative care: the development of the AmiPal podcast. BMJ Supportive \& Palliative Care, 7(2), 212-217. Recuperado de doi: 10.1136/bmjspcare-2016-001140

Paladino-Christin, M., \& Betrancourt, M. (2016). Uses and design preferences for professional 50 audio podcasts: survey of ICT professionals. International Journal of Technologies in Higher Education, 13(2), 46-59. Recuperado de https://doi.org/10.18162/ritpu-2016-v13n2-04

Patrick, M. D., Stukus, D. R., \& Nuss, K. E. (2019). Using podcasts to deliver pediatric educational content: Development and reach of PediaCast CME. DIGITAL HEALTH, 5, 1-9. Recuperado de https://doi.org/10.1177/2055207619834842

Pinto-Llorente, A. M., Sanchez-Gomez, M. C., Garcia-Penalvo, F. J., \& Cabezas-Gonzalez, M. (2017). Blended learning modality and English pronunciation: Findings from an ICT-supported model. RED. Revista de Educación a Distancia, (52). Recuperado de http://dx.doi.org/10.6018/red/52/4

Purdy, E., Thoma, B., Bednarczyk, J., Migneault, D., \& Sherbino, J. (2015). The use of free online educational resources by Canadian emergency medicine residents and program directors. Canadian Journal of Emergency Medicine, 17(2), 101-106. Recuperado de doi: $10.1017 /$ cem.2014.73

Rendón-López, L. M., \& Ortega-Carrillo, J. A. (2018). Evaluación de la calidad de programas de radio universitaria / Quality evaluation of university radio programs. Revista Mediterránea de Comunicación / Mediterranean Journal of Communication, 9(1), 151-175. Recuperado de https://www.doi.org/10.14198/MEDCOM2018.9.1.10

Riddell, J., Swaminathan, A., Lee, M., Mohamed, A., Rogers, R., \& Rezaie, S. R. (2017). A

55 Survey of Emergency Medicine Residents' Use of Educational Podcasts. Western Journal of Emergency Medicine, 18(2), 229-234. Recuperado de doi: 10.5811/westjem.2016.12.32850

Santos, S., Brites, M. J., Jorge, A., Catalão, D., \& Navio, C. (2015). Learning for life: A case study on the development of online community radio. Cuadernos.Info, (36), 111-123. Recuperado de doi: 10.7764/cdi.36.610

Sauter, T. C., Exadaktylos, A., Krummrey, G., Lehmann, B., Brodmann-Maeder, M., \& Hautz, W. E. (2018). Development, implementation and first insights of a time-and locationindependent longitudinal postgraduate curriculum in emergency medicine. GMS Journal for Medical Education, 35(4), 1-14. Recuperado de https://dx.doi.org/10.3205\%2Fzma001190

58 Schmidt, H. E. (2016). Scientific, technical, and medical podcasting in Korea. Science Editing, 3(1), 43-48. Recuperado de doi: 10.6087/kcse.62

Seena, S. T., \& Pillai Sudhier, K. G. (2014). Impact of web 2.0 technology applications in

59 kerala university library: Library professionals' perspective. DESIDOC Journal of Library and Information Technology, 34(5), 419-425. Recuperado de doi: 10.14429/djlit.34.6784

Sejdiu, S. (2017). Are listening skills best enhanced through the use of multimedia technology. Digital Education Review, (32), 60-72. Recuperado de https://doi.org/10.1344/der.2017.32.60-72

Semakula, D., Nsangi, A., Oxman, M., Austvoll-Dahlgren, A., Rosenbaum, S., Kaseje, M., Nyirazinyoye, L., Fretheim, A., Chalmers I., Oxman, A. D., \& Sewankambo, N. K. (2017). Can

61 an educational podcast improve the ability of parents of primary school children to assess the reliability of claims made about the benefits and harms of treatments: study protocol for a randomised controlled trial. Trials, 18(1), 31. Recuperado de doi: 10.1186/s13063-016-1745-y Sevilla, J. E. (2018). Integrating podcasts in the EFL classroom. A case study in $1^{\circ}$ E.S.O. / Integrando podcasts en el aula de inglés como lengua extranjera. Estudio de caso en $1^{\circ}$ de E.S.O. Complutense Journal of English Studies, 26, 193-217. Recuperado de http://dx.doi.org/10.5209/CJES.58382

62 E.S.O. Complutense Journal of English Studies, 26, 193-217. Recuperado de
Shekhavtsova, S. O. (2019). Organization of distance subject-subject interaction in the course of

63 future english philologists training. Information Technologies and Learning Tools, 71(3), 236247. Recuperado de https://doi.org/10.33407/itlt.v71i3.2459

Shishkovskaya, J. V., \& Sokolova, E. Y. (2015). Integration of Web 2.0 technologies into the

64 process of students' self-directed English learning. Mediterranean Journal of Social Sciences, 6(2), 541-545. Recuperado de doi: 10.5901/mjss.2015.v6n2p541

Smith, K., \& Morris, N. P. (2014). Evaluation of biomedical science students use and 65 perceptions of podcasting. Bioscience Education, 22(1), 3-15. Recuperado de doi: 10.11120/beej.2014.00024 
Synorub, H. P., Nestayko, I. M., Poplavska, I. V., Medynska, O. Y., \& Poplavska, N. M. (2019). Crossmedia as an innovative technology in training the humanities students in higher school. Information Technologies and Learning Tools, 70(2), 257-270. Recuperado de https://doi.org/10.33407/itlt.v70i2.2762

Thoma, B., Chan, T. M., Paterson, Q. S., Milne, K., Sanders, J. L., \& Lin, M. (2015).

67 Emergency Medicine and Critical Care Blogs and Podcasts: Establishing an International Consensus on Quality. Annals of Emergency Medicine, 66(4), 396-402. Recuperado de doi: 10.1016/j.annemergmed.2015.03.002

Thoma, B., Chan, T. M., Desouza, N., \& Lin, M. (2015). Implementing peer review at an

68 emergency medicine blog: bridging the gap between educators and clinical experts. Canadian Journal of Emergency Medicine, 17(2), 188-191. Recuperado de doi: $10.2310 / 8000.2014 .141393$

Thoma, B., Murray, H., Huang, S. Y. M., Milne, W. K., Martin, L. J., Bond, C. M., Mohindra,

69 R., Chin, A., Yeh, C. H., Sanderson, W. B., \& Chan, T. M. (2018). The impact of social media promotion with infographics and podcasts on research dissemination and readership. Canadian Journal of Emergency Medicine, 20(2), 300-306. Recuperado de doi: 10.1017/cem.2017.394

Thoma, B., Sanders, J. L., Lin, M., Paterson, Q. S., Steeg, J., \& Chan, T. M. (2015). The Social

70 Media Index: Measuring the Impact of Emergency Medicine and Critical Care Websites. Western Journal of Emergency Medicine, 16(2), 242-249. Recuperado de doi: 10.5811/westjem.2015.1.24860

Thurtle, N., Banks, C., Cox, M., Pain, T., \& Furyk, J. (2016). Free Open Access Medical Education resource knowledge and utilisation amongst Emergency Medicine trainees: A survey in four countries. African Journal of Emergency Medicine, 6(1), 12-17. Recuperado de https://doi.org/10.1016/j.afjem.2015.10.005

72 Ting, K. -Y. (2014). Blended learning as a theoretical framework for the application of

72 podcasting. English Language Teaching, 7(5), 128-135. Recuperado de doi: 10.5539/elt.v7n5p128

van Wyk, M. M. (2017). Exploring student teachers' views on eportfolios as an empowering

73 tool to enhance self-directed learning in an online teacher education course. Australian Journal of Teacher Education, 42(6), 1-21. Recuperado de doi: 10.14221/ajte.2017v42n6.1

Veloso, M. L. B., \& Páez, J. J. P. (2018). Emisoras infantiles en internet. Análisis de una oferta

74 alternativa para el niño-oyente / Radio stations for children on the internet. Analysis of an alternative offer for the child-listener. ICONO 14, Revista de comunicación y tecnologías emergentes, 16(2), 100-120. Recuperado de https://doi.org/10.7195/ri14.v16i2.1180

Vogelsang, M., Rockenbauch, K., Wrigge, H., Heinke, W., \& Hempel, G. (2018). Medical

75 Education for "Generation Z": Everything online?!-An analysis of Internet-based media use by teachers in medicine. GMS journal for medical education, 35(2), 1-20. Recuperado de https://dx.doi.org/10.3205\%2Fzma001168

Wojciechowska, B., \& Wilczyńska, W. (2017). Interactive procedures in meaning construction as models in developing complex foreign language oral competences [Procédés interactifs de

76 construction du sens dans le discours. Un modèle dans le développement des compétences orales au niveau avancé en langue étrangère ?]. Studia Romanica Posnaniensia, 44(2), 67-82. Recuperado de doi: 10.14746/strop.2017.442.004

Zaver, F., Craddick, M., Sanford, A., Sefa, N., Hughes, G., \& Lin, M. (2017). ALiEM Blog and

77 Podcast Watch: Toxicology. Western Journal of Emergency Medicine, 18(6), 1114-1119. Recuperado de doi: 10.5811/westjem.2017.6.33952

Zaver, F., Hansen, M., Leibner, E., Little, A., \& Lin, M. (2016). Blog and Podcast Watch:

78 Pediatric Emergency Medicine. Western Journal of Emergency Medicine, 17(5), 513-518. Recuperado de doi: 10.5811/westjem.2016.6.30193

79 Zetie, K. P. (2018). Extending student knowledge and interest through super-curricular activities. Physics Education, 53(2). Recuperado de doi: 10.1088/1361-6552/aa98c0

Zhigadlo, V. E., \& Odinokaya, M. A. (2017). Usage of training podcasts technology in the

80 interactive learning process of hindi language in Technical University as the basis of Upbringing process in supplementary humanitarian education. Yazyk I Kultura - Language and Culture, (38), 207-226. Recuperado de http://dx.doi.org/10.17223/19996195/38/14

Zoidl, P., Sacherer, F., Heschl, S., Steiner, K., Zajic, P., \& Prause, G. (2018). Nonsense or highquality advanced training?: Thoughts on FOAM [Schaumschlägerei oder Qualitätsfortbildung?: Gedanken zu FOAM]. Notfall Und Rettungsmedizin, 21(4), 317-319. Recuperado de doi: 10.1007/s10049-017-0317-3

Fuente: elaboración propia. 
La base de datos de acceso público con los 81 artículos incluidos en la muestra final se encuentra disponible en (https://bit.ly/2yLSaoF).

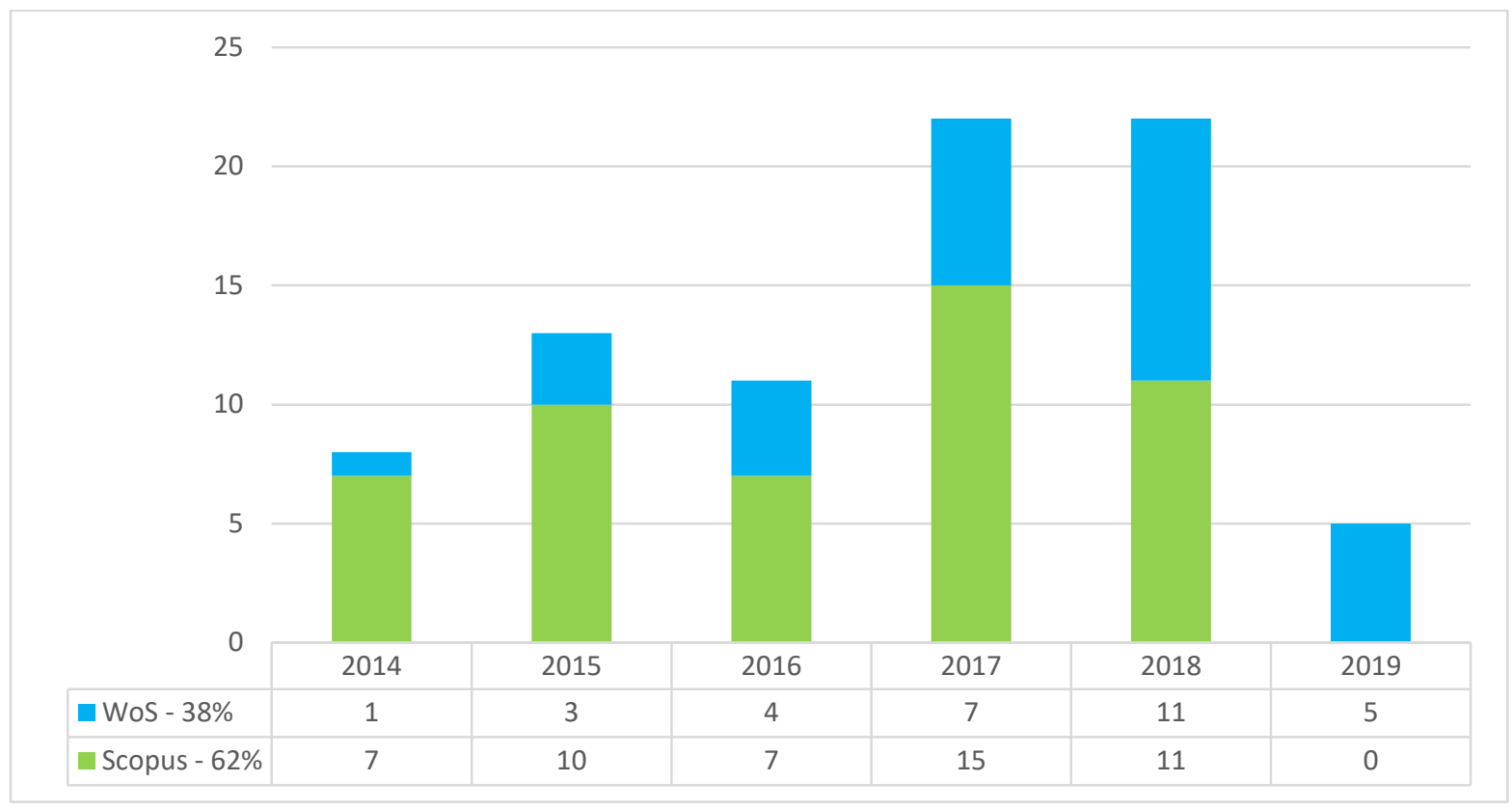

Figura 2: artículos de acceso abierto en función del año de publicación en las bases de datos Scopus y WoS.

Fuente: elaboración propia.

La relación de publicaciones en función del año de publicación (figura 2) sigue una tendencia estable a lo largo del tiempo, ligeramente ascendente. Los años con más publicaciones fueron 2017 y 2018 (ambos con 22 artículos) mientras que 2014 y 2016 fueron los años en los que menos se publicó (8 y 11 artículos respectivamente). Los datos de 2019 (5 artículos) están condicionados por la fechas de la búsqueda, febrero de ese año, por lo que no se han de tener en cuenta en el análisis bibliométrico.

Atendiendo a las bases de datos, Scopus cuenta con 50 artículos (62\%) y WoS con 31 (38\%). Resulta llamativo cómo desciende el número total de ítems finales al añadir el filtro de solo artículos de acceso abierto. La razón es que hay menos revistas de acceso abierto disponibles.

Posteriormente, se analizaron los títulos y los abstract de los artículos para obtener la respuesta a las RQ restantes. En algún caso, cuando el resumen no ofreció la suficiente información, se consultó el texto completo.

RQ1.1 - ¿Qué métodos de investigación se aplicaron en los estudios?

Los resultados muestran que la mayoría de estudios analizados aplicaron métodos empíricos, 43 en su vertiente cuantitativa y otros 24 mixta. Además, se registraron 14 artículos de corte teórico. En la tabla 4 se incluyen los artículos clasificados según el método de investigación utilizado y su porcentaje sobre el total de la muestra. 
RLCS, Revista Latina de Comunicación Social, 77, 179-201

[Investigación] DOI: 10.4185/RLCS-2020-1454 | ISSN 1138-5820| Año 2020

Tabla 4. Métodos de investigación que aplicaron los estudios.

\begin{tabular}{llll}
\hline Método & Ref. & Total & $\begin{array}{l}\text { Porcentaje } \\
(\mathbf{N}=\mathbf{8 1})\end{array}$ \\
\hline Teórico & $4,6,19,22,23,46,57,58,60,63,66,67,74,81$ & 14 & $17 \%$ \\
\hline \multirow{2}{*}{ Cuantitativo } & $1,5,7,9,10,11,20,27,28,29,30,31,32,33,35$, & & \\
& $36,37,38,40,41,43,44,45,47,49,50,51,52,53$, & 43 & $53 \%$ \\
\hline \multirow{2}{*}{ Mixto } & $54,55,59,61,64,65,68,69,70,71,75,77,78,79$ & & \\
& $2,3,8,12,13,14,15,16,17,18,21,24,25,26,34$, & 24 & $30 \%$ \\
\hline
\end{tabular}

Fuente: elaboración propia.

RQ1.2 - ¿Cuál es la distribución geográfica de los autores que más publicaron en el área?

De los 81 artículos analizados, los países que cuentan con más publicaciones son Estados Unidos (19), Canadá (12), Reino Unido (7), España (7) y Brasil (5) respectivamente. Se puede apreciar en la figura 3 que Norteamérica concentra gran parte del total publicado. El criterio que se utilizó para establecer el origen geográfico fue la procedencia de la universidad a la que estuviera afiliado el primer autor de cada artículo.

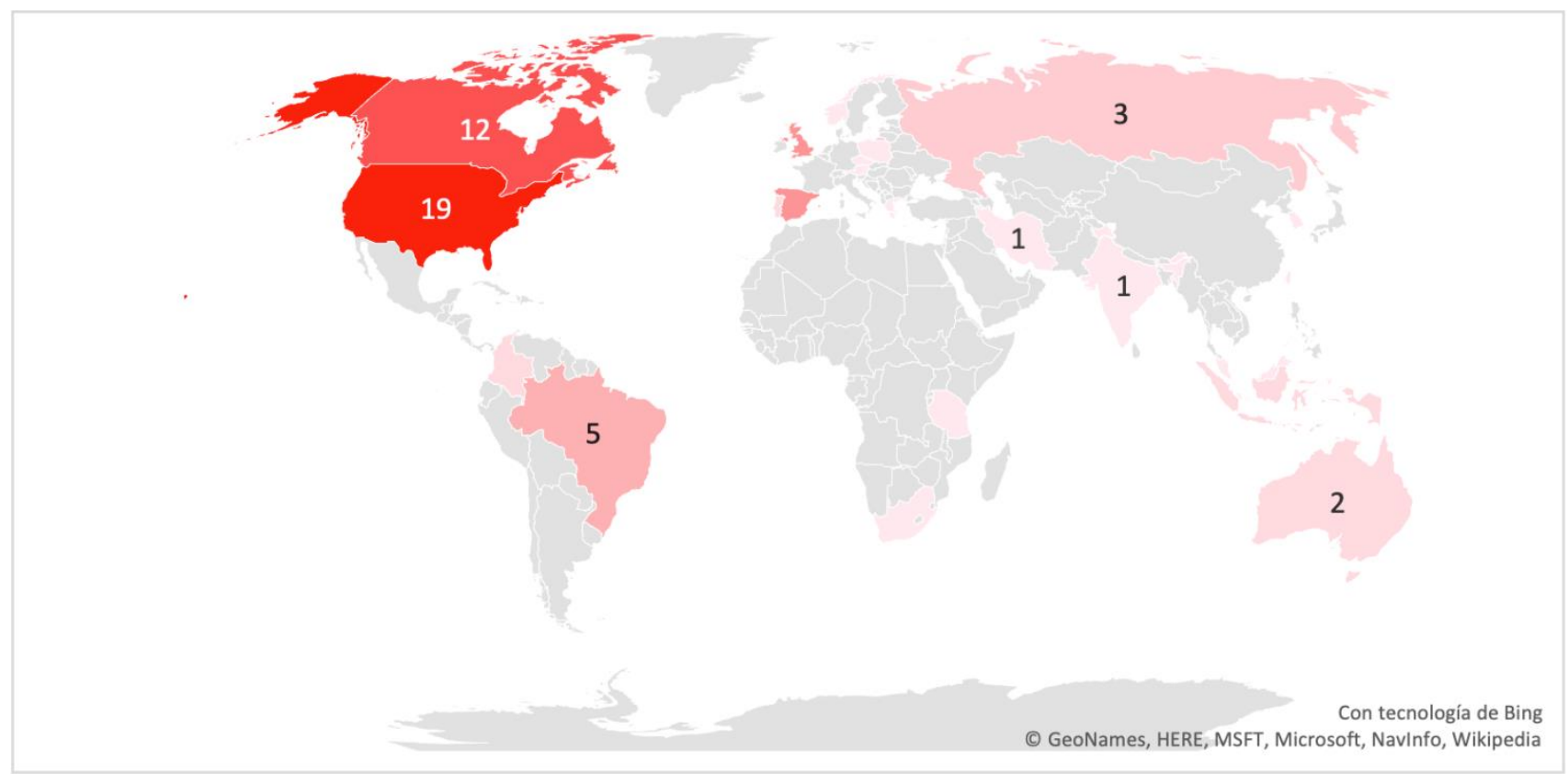

Figura 3: cantidad de publicaciones por países.

Fuente: elaboración propia.

RQ1.3 - ¿Cuáles son los artículos más citados?

Los artículos sumaron un total de 345 citas. Atendiendo a los cuartiles según el índice de impacto de las revistas, la muestra de artículos se distribuye de la siguiente manera: 18 artículos en Q1, 20 en Q2, 13 en Q3 y 4 en Q4. Se registraron otros 26 artículos procedentes de la base de datos Emerging Sources Citation Index (ESCI) de WoS. Como indica la tabla 5, un 47\% del total de estudios se publicaron en los dos primeros cuartiles. 
Tabla 5. Clasificación de artículos según el cuartil de su revista.

\begin{tabular}{llll}
\hline Cuartil & Ref. & Total & $\begin{array}{l}\text { Porcentaje } \\
(\mathbf{N}=\mathbf{8 1})\end{array}$ \\
\hline Q1 & $5,6,14,27,28,32,38,39,41,43,44,46,47,55,61,68,69,77$ & 18 & $22 \%$ \\
\hline Q2 & $\begin{array}{l}2,7,9,10,12,16,18,29,31,33,35,42,48,49,53,67,70,72, \\
73,78\end{array}$ & 20 & $25 \%$ \\
\hline Q3 & $1,3,4,8,19,21,45,56,59,64,76,79,81$ & 13 & $16 \%$ \\
\hline Q4 & $13,36,58,65$ & 4 & $5 \%$ \\
\hline ESCI & $\begin{array}{l}11,15,17,20,22,23,24,25,26,30,34,37,40,50,51,52,54, \\
57,60,62,63,66,71,74,75,80\end{array}$ & 26 & $32 \%$ \\
\hline
\end{tabular}

Fuente: elaboración propia.

Para responder con mayor concisión a la RQ1.3, se identificaros aquellos artículos que contaran con 10 o más citas, que se consideraron de alto impacto. Se representaron jerárquicamente en la figura 4 , atendiendo a criterios de cantidad de citas y peso relativo en los diferentes cuartiles de impacto.

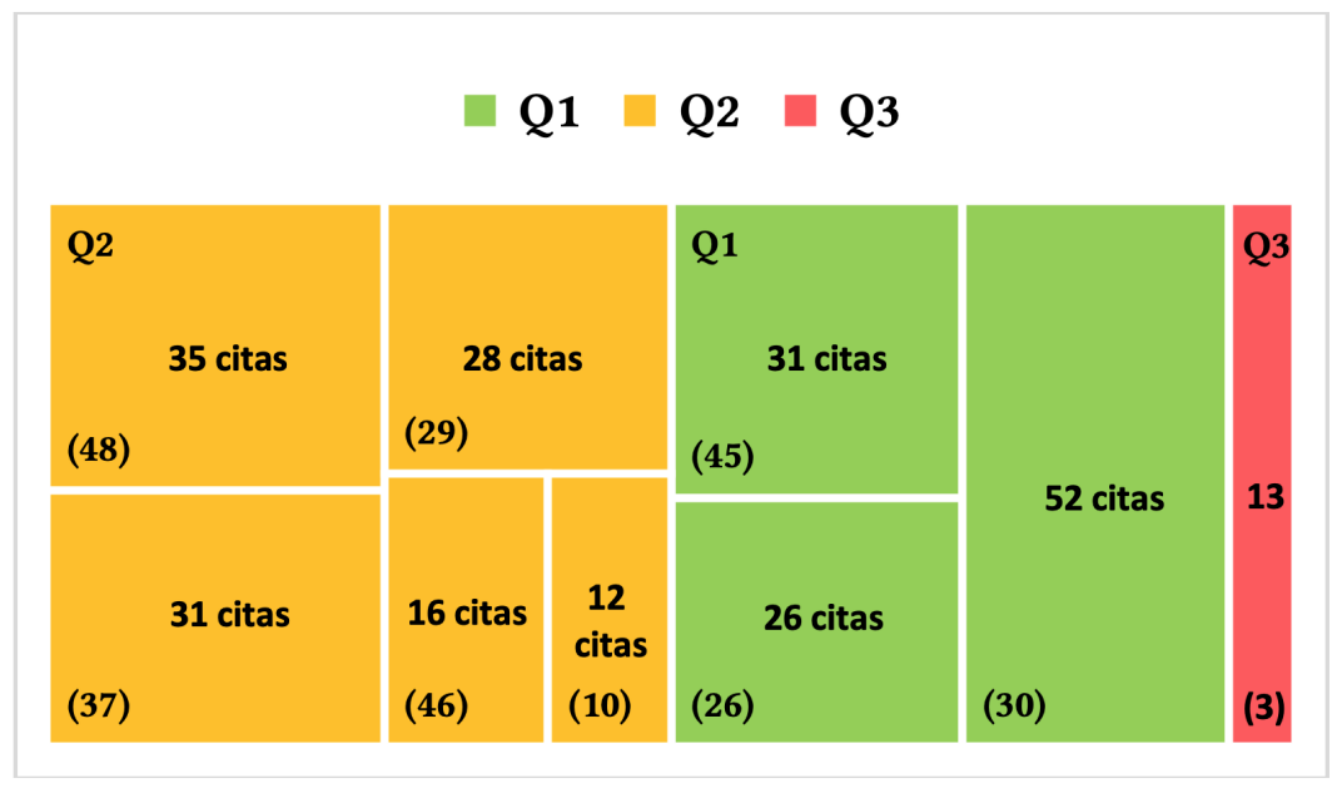

Figura 4: artículos con más de 10 citas ordenados conforme a su cuartil.

Fuente: elaboración propia.

La mayoría de artículos con más citas se situaron en el Q2, con un total 5 artículos y 122 citas en total. El Q1 es el segundo más importante, con 3 artículos de alto impacto que sumaron 109 citas. En el Q3 solo se encontró 1 artículo de alto impacto, con 13 citas en total. No se halló ningún artículo con más de 10 citas perteneciente al Q4 o a ESCI.

RQ4 - ¿Cuáles son las revistas que más publicaron sobre la temática?

Se identificaron 8 revistas con más de un artículo publicado (tabla 6), que aglutinan el 35\% de toda la muestra estudiada. En cuanto al área temática, pueden dividirse entre revistas de educación en medicina (19 artículos) y las de tecnología educativa (6 artículos). Destacan por número de 
publicaciones las revistas Western Journal of Emergency Medicine (10) y Canadian Journal of Emergency Medicine (4), ambas pertenecientes al ámbito médico.

Tabla 6. Revistas con más de dos publicaciones.

\begin{tabular}{|c|c|c|c|c|}
\hline Revista & Cuartil & Ref. & Total & $\begin{array}{l}\text { Porcentaje } \\
(\mathrm{N}=\mathbf{8 1})\end{array}$ \\
\hline $\begin{array}{l}\text { Western Journal of Emergency } \\
\text { Medicine }\end{array}$ & Q2 & $\begin{array}{l}7,10,18,19,20,22,38,48, \\
52,53\end{array}$ & 10 & $12 \%$ \\
\hline $\begin{array}{l}\text { Canadian Journal of Emergency } \\
\text { Medicine }\end{array}$ & Q1 & $28,37,46,47$ & 4 & $5 \%$ \\
\hline CUREUS & ESCI & $11,37,40$ & 3 & $4 \%$ \\
\hline $\begin{array}{l}\text { Information Technologies and } \\
\text { Learning Tools }\end{array}$ & ESCI & $34,63,66$ & 3 & $4 \%$ \\
\hline $\begin{array}{l}\text { Australian Journal of Teacher } \\
\text { Education }\end{array}$ & Q2 & 14,50 & 2 & $2,5 \%$ \\
\hline Educational Technology \& Society & $\mathrm{Q} 2$ & 23,35 & 2 & $2,5 \%$ \\
\hline $\begin{array}{l}\text { International Review of Research } \\
\text { in Open and Distributed Learning }\end{array}$ & Q1 & 6,21 & 2 & $2,5 \%$ \\
\hline $\begin{array}{l}\text { Journal of Medical Internet } \\
\text { Research }\end{array}$ & Q1 & 26,31 & 2 & $2,5 \%$ \\
\hline
\end{tabular}

Fuente: elaboración propia.

RQ2 - ¿En qué contextos se desarrollaron los artículos?

Para responder a la RQ2, se utilizó la clasificación de Ramírez-Montoya y García-Peñalvo (2018), que clasifican los contextos de actuación educativa en académico, social, empresarial y cultural. Así, se analizaron los artículos atendiendo al contexto en el que se había realizado la investigación.

Concretamente, se hallaron 35 artículos en el contexto académico, 10 en el social, 9 en el empresarial y 1 relativo al contexto cultural. En la tabla 7 se muestra la relación de los artículos en función de cada uno de los contextos, mientras que la figura 5 clasifica los estudios en contexto académico atendiendo a la etapa educativa específica en la que se engloban.

Tabla 7. Contextos de los estudios.

\begin{tabular}{llll}
\hline Contexto & Ref. & Total & $\begin{array}{l}\text { Porcentaje } \\
(\mathbf{N}=\mathbf{8 1})\end{array}$ \\
\hline & $1,3,5,6,7,10,11,13,16,17,18,19,20,21,22,23$, & \\
Académico & $\begin{array}{l}24,25,26,30,31,33,34,35,36,37,39,40,41,42,45, \\
47,48,50,52,54,58,59,60,61,62,63,64,65,66,67,\end{array}$ & $72 \%$ \\
& $69,70,71,72,73,75,76,77,78,79,80,81$ & \\
\hline Social & $4,8,12,14,38,46,49,51,56,68,74$ & 11 & $13 \%$ \\
\hline Empresarial & $9,27,28,29,32,43,44,53,55,57$ & 10 & $12 \%$ \\
\hline Cultural & 2,15 & 2 & $2 \%$ \\
\hline
\end{tabular}

Fuente: elaboración propia. 


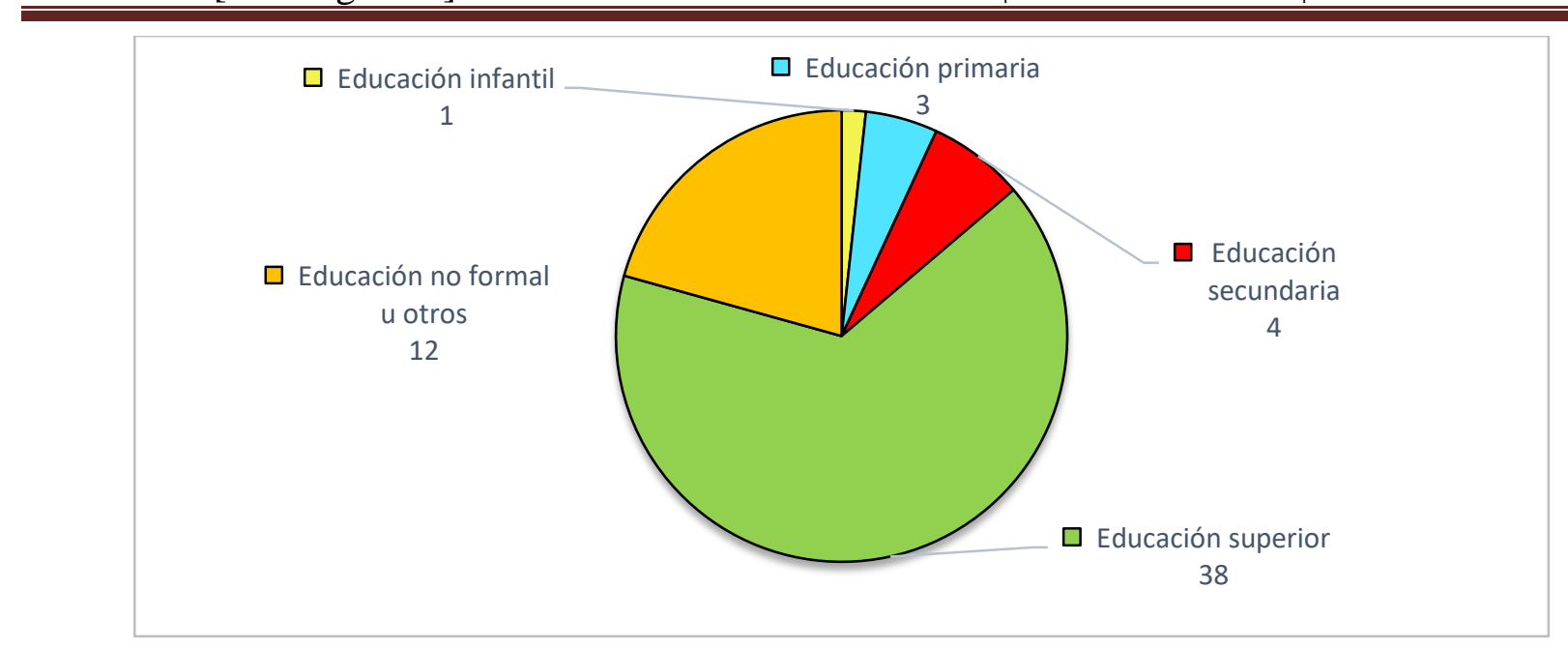

Figura 5: distribución de artículos en contexto académico según su etapa educativa.

Fuente: elaboración propia.

RQ3 - ¿Cuáles son las categorías temáticas en las que se estructuró el uso educativo del podcast de audio?

Por último, la RQ3 identificó las categorías emergentes según los enfoques más concretos de los estudios, agrupándolos según la función educativa que confieren al podcast (producción, selección/uso, diseminación y movilización).

La tabla 8 muestra cómo conciben los estudios el podcasting según la taxonomía de prácticas educativas abiertas y el peso porcentual de cada categoría. De acuerdo con lo expuesto, y considerando el formato podcast como REA, se encontraron 23 artículos sobre producción (28\%), 30 artículos sobre selección/uso (37\%), 3 artículos sobre diseminación (4\%) y 25 artículos centrados en movilización (31\%).

Tabla 8. Estudios según su función educativa.

\begin{tabular}{|c|c|c|}
\hline Categoría & Total & $\begin{array}{l}\text { Porcentaje } \\
(\mathbf{N}=\mathbf{8 1})\end{array}$ \\
\hline Producción & $\begin{array}{l}1,5,11,16,17,21,25,33,34,36,37,38,42,44,23 \\
46,49,50,54,58,62,68,70,81\end{array}$ & $28 \%$ \\
\hline Selección/Uso & $\begin{array}{l}6,9,10,13,18,19,20,22,24,27,28,29,30,32 \\
35,40,43,52,53,55,57,60,63,65,66,67,72,75,30 \\
77,78\end{array}$ & $37 \%$ \\
\hline Diseminación & $41,51,69$ & $4 \%$ \\
\hline Movilización & $\begin{array}{l}2,3,4,7,8,12,14,15,23,26,31,39,45,47,48, \\
56,59,61,64,71,73,74,76,79,80\end{array}$ & $31 \%$ \\
\hline
\end{tabular}

Fuente: elaboración propia.

\section{Discusión y conclusiones}

La muestra de artículos estudiada dibuja una tendencia constante de publicación desde 2014 hasta la actualidad como se muestra en la figura 2. La mitad de los artículos se encuentra en los cuartiles 
superiores de impacto, Q1 y Q2 (tabla 5), más aún los más citados (figura 4). Los estudios presentan un enfoque práctico de la temática: aplican métodos empíricos en su mayoría, tanto cuantitativos como mixtos (tabla 4). La tendencia hacia investigaciones empíricas se asemeja a la del estudio de Hew (2009). La encuesta y la entrevista fueron los métodos más utilizados en los estudios empíricos, junto a otros como el estudio de caso. En cuanto a regiones, Estados Unidos, Canadá, Reino Unido, España y Brasil fueron los países que más artículos publicaron sobre la temática (figura 3). No es de extrañar que Estados Unidos y Canadá aparezcan en primer lugar, dado que estudios de medios como el de Edison Research (2019) ya evidenciaban que Norteamérica es la región donde mayor presencia tiene el podcasting a nivel mundial.

Por otro lado, cabe destacar que la mayoría de la producción científica se centra en contextos académicos, concretamente centros de educación formal, aunque también en menor medida en contextos sociales y empresariales (tabla 7). Sin embargo, como muestra la figura 5, es en la etapa de educación superior donde se ha concentrado el grueso de la investigación. McGarr (2009) distingue entre tres categorías respecto a la implementación de podcasts en educación superior: sustitutivo y complementario de las lecciones, o bien creativo, lo cual implicaría que los alumnos crearan los contenidos a partir de lo aprendido. Por su parte, Forbes y Khoo (2015) encontraron que los podcasts generados por los estudiantes tenían potencial desde el punto de vista de la evaluación formativa a distancia, así como para compartir ideas entre alumnos y docentes. No obstante, atendiendo a la creciente complejidad de las categorías mencionadas, lo más habitual en la literatura se concentra en las categorías sustitutiva y complementaria, siendo la producción creativa más costosa y, por tanto, escasa.

Respecto a los usos concretos del formato podcast, o categorías que emergen de los estudios, se encontró que la literatura incide en lo relativo a la producción, selección y uso de los recursos de podcasts y su movilización para fines educativos (tabla 8). El espectro de usos educativos ha oscilado en el pasado entre el aprovechamiento de los contenidos del podcast a modo de divulgación en universidades y centros educativos (McGarr, 2009; Lomicka \& Lord, 2011; de-Lara-González \& delCampo-Cañizares, 2018, p. 351), y una aplicación del propio formato como vehículo para impartir formación en modo no presencial (Hasan \& Hoon, 2013; Forbes \& Khoo, 2015). El mapeo realizado descubre un variado elenco de propuestas en torno al podcast que los investigadores llevaron a cabo en los últimos 5 años: estudian el podcasting en entornos de educación formal en sus diversas etapas educativas; pero también en el ámbito institucional, en lo relativo a la formación del personal. Los usos son muy variados, siendo los principales la enseñanza de idiomas, el entrenamiento de personal médico, la alfabetización digital, y la educación social y sanitaria en contextos informales. También se documenta -en menor medida- el papel del podcast como medio difusor de la producción científica, lo cual refiere a su faceta de instrumento para la diseminación (Ramírez-Montoya y Burgos, 2012). A la luz de lo expuesto, se aprecia que el interés por el podcast educativo no solo sigue vigente, sino que, a juzgar por los resultados, sus usos van evolucionando con el tiempo.

Este mapeo sistemático encuentra su principal limitación en la selección del objeto de estudio. Por un lado, se resolvió limitar el concepto de podcast a aquel formato consistente en archivos digitales sindicados en formato de audio, puesto que como se explicó en la parte introductoria, este trabajo considera que podcast y vodcast son conceptos con características diferenciables. En consecuencia, todos aquellos estudios que se refieren a podcast de vídeo fueron descartados. Por otro lado, se limitó la búsqueda a estudios publicados en acceso abierto atendiendo a criterios de accesibilidad y oportunidad (tabla 2), renunciando conscientemente a otros muchos artículos de calidad que hubieran podido ser interesantes para la revisión. Además, la naturaleza de un mapeo sistemático implica per se una limitación de profundidad en el análisis. Se trata de presentar la temática de forma más descriptiva que analítica. También, limitar la búsqueda a los últimos 5 años deja fuera trabajos 
anteriormente publicados. Sin embargo, esta es una decisión deliberada en el diseño de esta investigación, al considerar cubierta esta etapa anterior por las revisiones de terceros autores citadas en la introducción.

De cara a superar las limitaciones mencionadas, se considera valioso para futuras investigaciones continuar ahondando en el podcast con fines educativos por medio de una revisión sistemática, que desgranaría la profundidad de los estudios localizados y los compararía entre sí. Adicionalmente, el presente mapeo detectó un déficit de estudios orientados hacia las etapas educativas de infantil, primaria y secundaria en la producción científica con más impacto (figura 5). Como hipótesis, esta carencia podría explicarse como consecuencia del perfil eminentemente práctico y no tanto investigador de muchos docentes de estas etapas educativas, los cuales acudirían a conferencias y congresos más que a revistas. Sin embargo, los profesionales de la educación superior estarían más familiarizados con el sistema de publicación científica. Así, animamos a los investigadores a estudiar lo que se publica en la denominada "literatura gris" podría arrojar luz sobre los usos del podcasting en estas etapas educativas mencionadas.

A partir de los estudios analizados concluimos que el podcast es un medio polivalente que se sigue utilizando de diversas maneras en educación: en su faceta de objeto educomunicativo, se ha utilizado para divulgar el conocimiento científico y social, instruir en entornos institucionales, así como fomentar actitudes críticas y reflexivas. Como REA, se ha aplicado en todas las etapas educativas, a distancia o en entornos presenciales, en contextos de educación formal, no formal e informal. Además, aludiendo a su facilidad de producción, se ha aprovechado para trabajar con comunidades de aprendizaje y en la elaboración de repositorios con contenidos formativos. El podcasting es una herramienta tecnológica más al servicio de la educación.

\section{Referencias}

Aguaded, I. (2005). Estrategias de edu-comunicación en la sociedad audiovisual. Comunicar, 12(24), 25-34. http://hdl.handle.net/10272/1208

Aguaded, I. (2011). La educación mediática, un movimiento internacional imparable La ONU, Europa y España apuestan por la educomunicación [Media education: an international unstoppable phenomenon UN, Europe and Spain support for edu-communication]. Comunicar, 18(37), 7-8. https://doi.org/10.3916/C37-2011-01-01

Aguaded, I. (2012). La educomunicación: una apuesta de mañana, necesaria para hoy. Aularia: Revista Digital de Comunicación, 1(2), 259-261. http://rabida.uhu.es/dspace/bitstream/handle/10272/6342/La_educomunicacion.pdf?sequence=2

Arribas, R. G., Gutiérrez, F. J. H., Fregoso, M. V., \& Arcos, C. A. M. (2018). Estudios sobre el podcast radiofónico: revisión sistemática bibliográfica en WOS y Scopus que denota una escasa producción científica. Revista Latina de Comunicación Social, (73), 1398-1411. http://dx.doi.org/10.4185/RLCS-2018-1313

Borges, F. (2009). Profcasts: aprender y enseñar con podcasts. Editorial UOC.

Blessinger, P., \& Bliss, T. (2016). Introduction to Open Education: Towards a Human Rights Theory. En Blessinger, P., \& Bliss, T. (Eds.), Open Education: International Perspectives in Higher Education (pp. 11-30). Open Book. http://www.jstor.org/stable/j.ctt1sq5v9n.6 
RLCS, Revista Latina de Comunicación Social, 77, 179-201

[Investigación] DOI: 10.4185/RLCS-2020-1454 | ISSN 1138-5820 | Año 2020

Brabazon, T. (2016). Digital dieting: From information obesity to intellectual fitness. Routledge.

Breugelmans, J. G., Roberge, G., Tippett, C., Durning, M., Struck, D. B., \& Makanga, M. M. (2018). Scientific impact increases when researchers publish in open access and international collaboration: A bibliometric analysis on poverty-related disease papers. PLOS ONE 13(9): e0203156. https://doi.org/10.1371/journal.pone.0203156

Bundy, A. (Ed.). (2004). Australian and New Zealand information leteracy framework, principles, standards and practice. Australian and New Zealand Institute for Information Literacy, 2. http://www.libnet.sh.cn/upload/htmleditor/File/130620025617.pdf

Burbules, N. C. (2012). El aprendizaje ubicuo y el futuro de la enseñanza [Ubiquitous Learning and the Future of Teaching]. Encounters, 13, 3-14.

https://ojs.library.queensu.ca/index.php/encounters/article/download/4472/4513/0

Burbules, N. C. (2014). El aprendizaje ubicuo: nuevos contextos, nuevos procesos. Entramados: educación y sociedad, 1(1), 131-134.

https://fh.mdp.edu.ar/revistas/index.php/entramados/article/view/1084/1127

CASCADE. (2012). Mapping in literature reviews.

https://as.exeter.ac.uk/media/universityofexeter/academicservices/educationenhancement/cascade/ Mapping_in_literature_reviews.pdf

Castells, M. (2006). The network society: From knowledge to policy. En Castells, M., \& Cardoso, G. (Eds.). The network society: From knowledge to policy (pp. 3-23). Johns Hopkins Center for Transatlantic Relations.

https://www.dhi.ac.uk/san/waysofbeing/data/communication-zangana-castells-2006.pdf

D’Antoni, S. (2009). Open Educational Resources: reviewing initiatives and issues. Open Learning: The Journal of Open, Distance and e-Learning, 24(1), 3-10.

https://doi.org/10.1080/02680510802625443

de-Lara-González, A., \& del-Campo-Cañizares, E. (2018). El podcast como medio de divulgación científica y su capacidad para conectar con la audiencia. Revista Mediterránea de Comunicación / Mediterranean Journal of Communication, 9(1), 347-359.

https://doi.org/10.14198/MEDCOM2018.9.1.15

Díaz, V. A. (2012). Pensar la sociedad de la información/conocimiento. Biblioteca Universitaria, 15(1), 35-47. http://dx.doi.org/10.22201/dgb.0187750xp.2012.1.40

Earp, S., Belanger, Y., \& O'Brien, L. (2006). Duke digital initiative end of year report. Duke University. https://learninginnovation.duke.edu/pdf/reports/ddiEval0506_final.pdf

Edison Research. (2019). The Infinite Dial 2019.

http://www.edisonresearch.com/wp-content/uploads/2019/03/Infinite-Dial-2019-PDF-1.pdf

EGM. (2017). $1^{a}$ ola EGM: el consumo de podcasts en España representa el 1,6\% de la escucha total de radio. https://www.aimc.es/a1mc-c0nt3nt/uploads/2017/05/170419_egm_2017ola1.pdf 
Esclapez, T. C. (2008). La enseñanza que no se ve: educación informal en el siglo XXI (Vol. 7). Narcea Ediciones.

Forbes, D., \& Khoo, E. (2015). Voice over distance: a case of podcasting for learning in online teacher education. Distance Education, 36(3), 335-350.

https://doi.org/10.1080/01587919.2015.1084074

Gallado, E. E. (2013). Competencia digital: revisión integradora de la literatura. Revista de Ciencias de la Educación Academicus, 1(3), 56-62. http://www.ice.uabjo.mx/media/15/2017/04/Art3 7.pdf

Gallego, J. I. (2010). Podcasting: distribución de contenidos sonoros y nuevas formas de negocio en la empresa radiofónica española (Tesis doctoral). Universidad Complutense de Madrid, Madrid. https://eprints.ucm.es/11205/1/T32070.pdf

García-Peñalvo, F. J. (2017). Mitos y realidades del acceso abierto. Education in the Knowledge Society, 18(1), 7-20. https://www.redalyc.org/pdf/5355/535554765001.pdf

Hammersley, B. (12 de febrero de 2004). Audible revolution. The Guardian. https://www.theguardian.com/media/2004/feb/12/broadcasting.digitalmedia

Hasan, M. M., \& Hoon, T. B. (2013). Podcast Applications in Language Learning: A Review of Recent Studies. English Language Teaching, 6(2), 128-135. https://files.eric.ed.gov/fulltext/EJ1076962.pdf

Hew, K. F. (2009). Use of Audio Podcast in K-12 and Higher Education: A Review of Research Topics and Methodologies. Educational Technology Research and Development, 57(3), 333-357. http://dx.doi.org/10.1007/s11423-008-9108-3

Jenkins, H., Purushotma, R., Werigel, M., Clinton, K., \& Robinson, A.J. (2009). Confronting the challenges of participatory culture: Media Education for the 21st century. The MIT Press.

Keegan, D. (2005). Mobile learning: the next generation of learning. Distance Education International, 36, 137-143. http://www.cin.ufpe.br/ mlearning/intranet/m-learning/book.doc

Khan, A. W. (2009). Foreward: Unesco. En Frau-Meigs, D., \& Torrent, J. (Eds.). Mapping media education policies in the world: visions, programmes and challenges. Grupo Comunicar. https://unesdoc.unesco.org/ark:/48223/pf0000181917.locale=es

Kitchenham, B., \& Charters, S. (2007). Guidelines for performing systematic literature reviews in software engineering. Keele University \& University of Durham. http://citeseerx.ist.psu.edu/viewdoc/download?doi=10.1.1.117.471\&rep=rep1\&type=pdf

Kitchenham, B., Pretorius, R., Budgen, D., Brereton, O. P., Turner, M., Niazi, M., \& Linkman, S. (2010). Systematic literature reviews in software engineering-a tertiary study. Information and software technology, 52(8), 792-805. https://doi.org/10.1016/j.infsof.2010.03.006

Kroll, J., Richardson, I., Prikladnicki, R., \& Audy, J. L. (2018). Empirical evidence in follow the sun software development: a systematic mapping study. Information and Software Technology, 93, 30-44. https://doi.org/10.1016/j.infsof.2017.08.011 
Lomicka, L., \& Lord, G. (2011). Podcasting-past, present and future: Applications of academic podcasting in and out of the language classroom. En Academic podcasting and mobile assisted language learning: Applications and outcomes (pp. 1-20). IGI Global.

McGarr, O. (2009). Podcasting in higher education: a review of the literature with particular reference to its influence on the traditional lecture. Australasian Journal of Educational Technology, 25(3), 309-321. https://doi.org/10.14742/ajet.1136

McKiernan, E. C., Bourne, P. E., Brown, C. T., Buck, S., Kenall, A., Lin, J., McDougall, D., Nosek, B. A., Ram, K., Soderberg, C. K., Spies, J. R., Thaney, K., Updegrove, A., Woo, K. H., \& Yarkoni, T. (2016). Point of view: How open science helps researchers succeed. Elife, 5. https://elifesciences.org/articles/16800

Melero, R. y Hernández-San-Miguel, J. (2014). Acceso abierto a los datos de investigación, una vía hacia la colaboración científica. Revista Española de Documentación Científica, 37(4). http://redc.revistas.csic.es/index.php/redc/article/view/869/1175

Naval, C., \& Arbués, E. (2015). Del uso de Internet en la promoción de virtudes sociales. Un caso concreto: Parlamento Cívico. Teoría De La Educación. Revista Interuniversitaria, 27(1), 33-52. http://revistas.usal.es/index.php/1130-3743/article/view/teoredu20152713352/13434

Naval, C., Serrano-Puche, J., Sádaba, C., y Arbués, E. (2016). Sobre la necesidad de desconectar: algunos datos y propuestas. Education in the Knowledge Society, 17(2), 73-90. http://hdl.handle.net/10171/41534

O'Reilly, T. (2005). What Is Web 2.0. O’Reilly Media Inc. http://goo.gl/HzTN3N

Paterson, Q. S., Thoma, B., Milne, W. K., Lin, M., \& Chan, T. M. (2015). A Systematic Review and Qualitative Analysis to Determine Quality Indicators for Health Professions Education Blogs and Podcasts. Journal Of Graduate Medical Education, 7(4), 549-554. https://doi.org/10.4300/JGMED-14-00728.1

Petersen, K., Vakkalanka, S., \& Kuzniarz, L. (2015). Guidelines for conducting systematic mapping studies in software engineering: An update. Information and Software Technology, 64, 1-18. https://doi.org/10.1016/j.infsof.2015.03.007

Phillips, F., Yu, C. Y., Hameed, T., \& El Akhdary, M. A. (2017). The knowledge society's origins and current trajectory. International Journal of Innovation Studies, 1(3), 175-191. https://doi.org/10.1016/j.ijis.2017.08.001

Piwowar, H., Priem, J., Larivière, V., Alperin, J. P., Matthias, L., Norlander, B., Farley, A., West, J., \& Haustein, S. (2018). The state of OA: a large-scale analysis of the prevalence and impact of Open Access articles. PeerJ, 6. https://doi.org/10.7717/peerj.4375

Ramírez-Montoya, M. S. (2018). Innovación abierta, interdisciplinaria y colaborativa para formar en sustentabilidad energética a través de MOOCs e investigación educativa. Education in the Knowledge Society (EKS), 19(4), 11-30. https://doi.org/10.14201/eks20171821932 
Ramírez-Montoya, M. S. y Burgos, J. V. (Coords.) (2012). Movimiento educativo abierto: Acceso, colaboración y movilización de recursos educativos abiertos. [eBook]. Lulú editorial digital. http://catedra.ruv.itesm.mx/handle/987654321/564

Ramírez-Montoya, M. S., \& García-Peñalvo, F. (2018). Co-creation and open innovation: Systematic literature review. [Co-creación e innovación abierta: Revisión sistemática de literatura]. Comunicar, 54, 09-18. https://doi.org/10.3916/C54-2018-01

Sellas, T. (2011). El Podcasting: la (r)evolución sonora. Editorial UOC.

Unesco. (2002). Forum on the impact of open courseware for higher education in developing countries: Final report. https://unesdoc.unesco.org/ark:/48223/pf0000128515.locale=es

Wang, X., Liu, C., Mao, W., \& Fang, Z. (2015). The open access advantage considering citation, article usage and social media attention. Scientometrics, 103(2), 555-564. https://link.springer.com/content/pdf/10.1007\%2Fs11192-015-1547-0.pdf

Zapata Ros, M. (2015). Teorías y modelos sobre el aprendizaje en entornos conectados y ubicuos. Bases para un nuevo modelo teórico a partir de una visión crítica del "conectivismo". Education in the Knowledge Society (EKS), 16(1), 69-102.

http://revistas.usal.es/index.php/eks/article/view/eks201516169102

\section{AUTORES:}

\section{Iñaki Celaya}

Maestro, pedagogo y máster en Investigación en Ciencias Sociales por la Universidad de Navarra. Personal Investigador en Formación y alumno del Programa de Doctorado en Educación y Psicología de la Universidad de Navarra, desde el año 2017. Su investigación se centra en la virtualidad educativa de los medios de comunicación digitales, concretamente el formato podcast de audio, que se enmarca en el Grupo de Investigación consolidado "Educación, ciudadanía y carácter" de la Universidad de Navarra. Colabora en la docencia en los grados de Educación y Psicología. Ha realizado una estancia de investigación en la Escuela de Humanidades y Educación del Tecnológico de Monterrey (México) de enero a junio de 2019.

icelaya@alumni.unav.es

Orcid ID: https://orcid.org/0000-0003-0384-3575

Google Scholar: https://bit.ly/2kicHus

\section{María Soledad Ramírez Montoya}

Doctora en Filosofía y ciencias de la Educación por la Universidad de Salamanca e investigadora titular de la Escuela de Humanidades y Educación del Tecnológico de Monterrey. Es directora de la Cátedra Unesco: "Movimiento educativo abierto para América Latina", directora de la oficina del International Council for Open of Distance Education (ICDE): OER Latin America, investigadora principal en la Red Strengthening Information Society Research Capacity Alliance (SIRCA), organizadora principal de la Red Latinoamericana Abierta Regional de Investigación Social y Educativa (CLARISE). Es miembro del Sistema Nacional de Investigadores en México. Sus líneas de investigación son las estrategias de enseñanza, los recursos tecnológicos para la educación, la formación de investigadores educativos y el movimiento educativo abierto. Su experiencia profesional contempla actividades docentes en todos los niveles educativos, decana de posgrados en humanidades y educación, direcciones de departamento, coordinadora de grupos de investigación y consultoría pedagógica. Es profesora investigadora invitada en programas doctorales de 
Universidades Europeas (Universidad de Salamanca, Universidad de Huelva y Universidad Internacional de La Rioja) y Universidades Latinoamericanas (Pontificia Universidad Católica del Perú, Universidad Técnica Particular de Loja, Universidad Católica del Perú y Universidad de la Sabana).

solramirez@tec.mx

Índice H: según GSM = 33; i10 = 105

Orcid ID: https://orcid.org/0000-0002-1274-706X

Google Scholar: https://bit.ly/2k4abrw

\section{Concepción Naval}

Catedrática y Decana de la Facultad de Educación y Psicología de la Universidad de Navarra. Su investigación se centra en la educación ciudadana y la participación social, el impacto social en los jóvenes de las tecnologías de la comunicación, la innovación educativa en la educación superior y la educación del carácter. Inició y dirige el Grupo de investigación consolidado en la Universidad de Navarra "Educación, ciudadanía y carácter" y el proyecto "Researching and promoting character education in Central and South American secondary schools". Templeton World Charity Foundation (2016-2019): https://bit.ly/2jZ5PBQ Dirige el Consejo Editorial de la revista "Estudios sobre educación" desde sus comienzos, en 2001 (Q2 en SJR, 2018). Es miembro del Editorial Board del "Journal of Social Sciences Education" (Alemania) y del Journal "Citizenship, Teaching and Learning"(UK); del Consejo Editorial de la "Revista Interuniversitaria de Formación del Profesorado", de la Revista "Sembrando Ideas" y de la "Revista Perspectiva Educacional" (Chile), entre otras. Ha sido Oliver Smithies Lecturer, Oxford University; Visiting Professor, Teachers College, Columbia University; Visiting Scholar Research, University of Harvard, University of Notre Dame (EEUU), Oficina Internacional de la Educación (Unesco); Profesora invitada, Universidad Panamericana, México; Fellow del Salzburg Seminar. Fue miembro del grupo de expertos del proyecto "A Study on Active Citizenship Indicators" financiado por la Comisión Europea, DG Education and Culture.

cnaval@unav.es

Índice H: según GSM = 22; i10 = 65

Orcid ID: https://orcid.org/0000-0002-5927-9398

Google Scholar: https://bit.ly/2jZkxsx

\section{Elena Arbués}

Profesora Contratada Doctora y Vicedecana de Alumnos de la Facultad de Educación y Psicología en la Universidad de Navarra. Su investigación se centra en la enseñanza de las ciencias experimentales en educación primaria, así como en la educación cívica y el fomento de la participación social. Ha sido Visiting Postdoctoral Scholar durante 2013 en Balliol College, Holywell Manor, University of Oxford. Es miembro de varios proyectos de investigación financiados en convocatorias públicas, actualmente es miembro investigador del proyecto "Aprendizaje-Servicio (APS) y empleabilidad de los estudiantes universitarios en España: competencias para la inserción laboral" (MINECO Plan Estatal (2017-2021) EDU2017-82629-R).

earbues@unav.es

Índice H: según $\mathrm{GSM}=7 ; \mathrm{i} 10=4$

Orcid ID: https://orcid.org/0000-0001-5872-830X

Google Scholar: https://bit.ly/21TKCK5 